\title{
Supragingival Plaque Removal with and without Dentifrice: A Randomized Controlled Clinical Trial
}

\author{
Fabricio B. ZANATTA ${ }^{1}$ \\ Raquel P. ANTONIAZZI² \\ Tatiana M. P. PINTO ${ }^{1}$ \\ Cassiano K. RÖSING ${ }^{3}$ \\ ${ }^{1}$ Graduate Program in Dentistry, UFSM - Federal University of Santa Maria, Santa Maria, RS, Brazil \\ ${ }^{2}$ Dental School, UNIFRA - Franciscan University Center, Santa Maria, RS, Brazil \\ ${ }^{3}$ Graduate Program in Dentistry, UFRGS - Federal University of Rio Grande do Sul, Porto Alegre, RS, Brazil
}

\begin{abstract}
The aim of this study was to compare the efficacy of dental plaque removal by brushing with and without conventional dentifrice. Twenty-four students aged 17 to 28 years participated in this randomized controlled clinical trial. Quadrants 1-3 or 2-4 were randomly allocated to the test group (brushing without dentifrice) or control group (brushing with dentifrice). After $72 \mathrm{~h}$ of cessation of oral hygiene, Quigley \& Hein (Turesky) plaque index was assessed before and after brushing by a calibrated and blind examiner. Overtime and intergroup comparisons were performed by Student's paired sample t-test at 5\% significance level. The results showed that both groups after toothbrushing presented statistically significant reductions in plaque, with no differences between them (from $3.06 \pm$ 0.54 to $1.27 \pm 0.26$ versus from $3.07 \pm 0.52$ to $1.31 \pm 0.23$ ). A separate analysis of the buccal and lingual aspects also showed no significant differences between groups. It may be concluded that the use of a conventional dentifrice during toothbrushing does not seem to enhance plaque removal capacity.
\end{abstract}

Key Words: toothbrushing, dental plaque, dentifrice, clinical trial, oral hygiene.

\section{INTRODUCTION}

Dental plaque has been defined as a complex community of bacterial species that is constantly formed over dental surfaces. Although this biofilm is exposed to saliva and other natural self-cleaning mechanisms, the removal of dental plaque is not naturally achieved (1). The role of dental plaque on the etiology of caries and periodontal diseases is well established in literature. Also, its mechanical removal by at-home and professional oral hygiene measures have been directly related to the prevention and treatment of caries and periodontal diseases $(2,3)$.

Regular oral hygiene is mandatory for dental plaque control. It is dependent on the individual's instruction and motivation and use of appropriate means. This way, within the available arsenal for controlling supragingival plaque, toothbrush, dental floss, interdental brushes, and end-tufted brushes among others are often used $(4,5)$. Toothbrush is the most used plaque control device. As adjuncts to toothbrushing, dentifrices and rinsing solutions have been proposed, in order to enhance the plaque removal efficacy (6-8).

The indication for dentifrices is mainly based on the presence of fluoride (9), antimicrobial agents that aim at further reducing plaque formation and/or removing a previously established plaque. Additionally, dentifrices are associated to sense of a pleasant flavor and coolness after usage. Dentifrices have also been used as plaque removal aids, especially because of their abrasive agents (10). The commonly used abrasive agents include silica, carbonates, alumina and more recently, perlite (11).

In addition to fluoride release and presence of antimicrobial agents, the actual adjunct role of dentifrices to mechanical removal of dental plaque is contradictory. Binney et al. (12) compared toothbrushing with dentifrices and with water did not find statistically significant differences between groups. Parizotto et al. (13) evaluated the efficacy of dental plaque removal by comparing conventional toothbrush with 
an end-tufted brush, with and without dentifrice There were no significant differences between both types of toothbrushes and the use of dentifrice did not contribute to dental plaque removal. Unfortunately, these results have not been conclusive due to a reduced experimental setting. Similarly, Danielsen et al. (14) compared in a crossover study, the additional effect on plaque removal with and without using dentifrices in children who brushed their teeth and chewed gum. Their results showed that the dentifrice did not have an additional effect on removing dental plaque. However, Almajed (15) found different results after comparing brushing using two types of brush with and without use of dentifrice. In this study higher reductions in plaque were found when a 2-head toothbrush was combined with dentifrice. Similar results have been reported previously (16).

This way, the impact of using a dentifrice as a mechanical agent to assist toothbrushing is still unclear. The null hypothesis of this trial is that dentifrice will not contribute to plaque removal by toothbrushing. The aim of this study was to compare the capacity of dental plaque removal by toothbrushing with and without the use of a conventional dentifrice.

\section{MATERIAL AND METHODS}

This study was a blind randomized controlled clinical trial with split-mouth design. Twenty-four systemically healthy students of both genders with mean age of $22.4 \pm 2.3$ years participated. Exclusion criteria comprised gingivitis (presence of gingival bleeding at any site); probing depth $>3 \mathrm{~mm}$ and/or attachment loss $>3 \mathrm{~mm}$ at any site; use of orthodontic appliance devices, dental prostheses, dental implants; presence of abrasions, restorations and/or carious lesions at cervical region; and natural dentition of less than 20 teeth.

This research protocol was approved by the Ethics Committee of the Franciscan University Center (CEPUNIFRA protocol \#058.2008.02) and the experimental procedures were in accordance with the applicable ethical standards on human experimentation and with the Helsinki Declaration of 1975, as revised in 2000. All participants read and signed an informed consent form prior to enrolment in the study.

\section{Sample Size Calculation}

Sample size estimates was based on the results of plaque reduction between the experimental groups of the study of Binney et al. (12), whose methodology was similar to those the present study. Alpha and beta errors of 0.05 and 0.2 were considered. Therefore, 24 participants were considered necessary.

\section{Dependent Variable}

The outcome of this study was evaluated in terms of presence of dental plaque. Quigley and Hein (17) (Q.H.) Index modified by Turesky, Gilmore and Glickman (18) was used in all participants, and recorded in an individual clinical file. The examiner was unaware of quadrant distribution.

\section{Reproducibility}

Prior to the start of the study, the examiner was trained by an experienced periodontist until reaching informal agreement on the diagnostic criteria. After that, calibration with 5 individuals who remained 3 days without mechanical plaque removal was performed. Double assessment of the index was performed with 1-h interval. Intra-examiner agreement resulted in a Kappa coefficient of 0.78 .

\section{Experimental Procedures}

Following selection, the participants received instructions about oral hygiene, and were submitted to a thorough dental prophylaxis for complete removal of dental plaque. Plaque retentive factors were also eliminated. The participants were instructed to interrupt any chemical and/or mechanical oral hygiene procedure for $72 \mathrm{~h}$. Then, dental plaque was stained by a $2 \%$ erythrosine solution (Replak; Dentsply Ind. e Com. Ltda., Petrópolis, RJ, Brazil), followed by a mouth rinsing with water for $1 \mathrm{~min}$. All erupted teeth, except for third molars, were examined by a blind examiner at 6 sites per tooth (mesiobuccal, mid-buccal, distobuccal, mesiolingual, mid-lingual, and distolingual surfaces).

Next, the set of dental quadrants (1-3 and 2-4) were randomly allocated to the experimental groups by the flip of a coin. One of the sets was brushed with multi-bristle brush (Tek Soft ${ }^{\circledR}$; Condor S/A, São Bento do Sul, SC, Brazil) without the use of dentifrice and the other set was brushed with multi-bristle brush using dentifrice (CloseUp White ${ }^{\circledR}$; Unilever Brasil Higiene e Limpeza Ltda, São Paulo, SP, Brazil). The brush head 
showed a round configuration with $10 \mathrm{~mm}$ long nylon filaments invested in a tuft hole with a diameter $1.6 \mathrm{~mm}$ and size of $2.5 \mathrm{~cm}$ height versus $1.0 \mathrm{~cm}$ width. Each head consisted of 3 rows of tuffs, with 9 tuffs per row, with a total of 43 tufts. There were approximately 23 filaments per tuft and each filament had a diameter of $200 \mu \mathrm{m}$. The form of endrounding was a filament with a rounded pointy end.

The percentage of abrasive in the dentifrice composition was $16.33 \%$ of silica. Sorbitol, aqua, sodium lauryl sulfate, aroma, cellulose gum, sodium fluoride $0.32 \%$, sodium saccharin and PEG-32 were the other components of dentifrice. The amount of dentifrice used was equivalent to half-length of the toothbrush's head.

Toothbrushing was performed by the individual's regular technique timed. Two minutes was used for each set, which corresponds to $1 \mathrm{~min}$ for each hemiarch, $30 \mathrm{~s}$ for buccal surfaces and $30 \mathrm{~s}$ for lingual/palatine surfaces. After toothbrushing, dental plaque was again disclosed and evaluated by the same examiner, who was blind to the quadrants brushed with or without dentifrice.

\section{Analysis of the Results}

Means and standard deviation of the Q. H. index of the two groups before and after toothbrushing were calculated. Also, separate mean values were calculated for buccal and palatal/lingual surfaces. Overtime (intragroup) and intergroup differences were compared by Student's paired sample t-test. The level of significance was set at 0.05 .

\section{RESULTS}

All 24 subjects who fulfilled all inclusion/ exclusion criteria completed the study and side effects were not observed. Figure 1 shows a flow diagram of the progress of clinical trial participants in the groups.

Both groups presented considerable dental plaque formation prior to toothbrushing. After brushing, both groups exhibited a statistically significant reduction $(p<0.05)$ in the mean plaque index, but no statistically significant differences $(p>0.05)$ were detected between groups (Table 1).

When the analysis was stratified into buccal and palatal/lingual surfaces, there were no statistically significant differences $(p>0.05)$ between quadrants using or not dentifrice, similarly to the whole mouth analysis. Palatal/lingual surfaces accumulated less plaque as compared with buccal surfaces at baseline. However, the final results demonstrate similar values for all surfaces. Thus, a higher mean reduction was obtained for buccal as compared with palatal/lingual surfaces $(70 \% v s .60 \%$, respectively). No significant differences were found

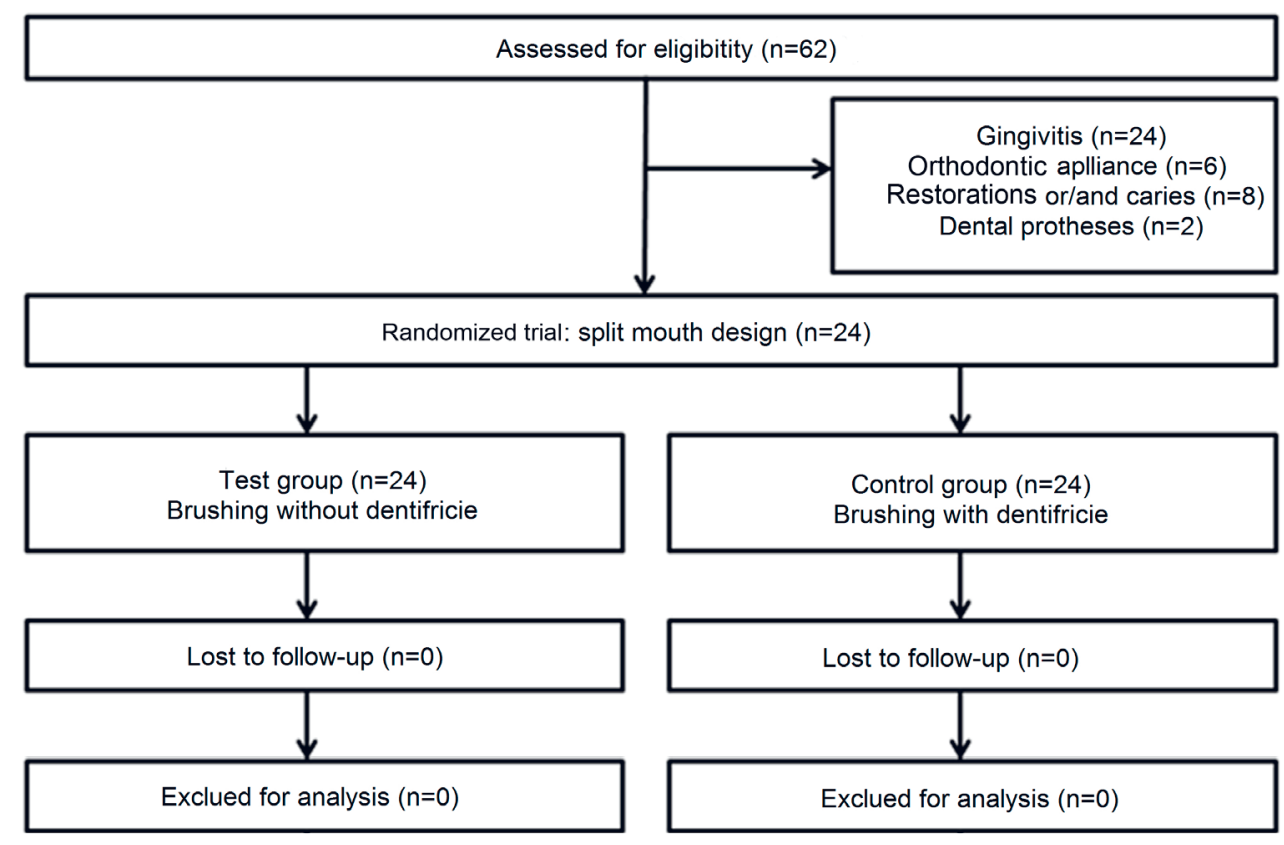

Figure 1. Flow diagram of the progress through the phases of the randomized clinical trial for the two groups. 
regarding the use or not of dentifrice (Table 2).

\section{DISCUSSION}

This study compared the capacity of plaque removal by toothbrushing with and without dentifrice. After toothbrushing, both groups showed a marked reduction in mean plaque index with no statistically significant differences between them. The experimental design used in this study (randomized controlled clinical trial) was chosen because it is the most adequate for such comparisons. The split-mouth approach allowed each participant being his/her own control. The fact that experimental groups were randomly divided into quadrants 1-3 or 2-4 enabled variables related to toothbrushing technique and force, aside form anatomical situations, to be randomly distributed in the groups as well. All participants were right-handed. The Quigley and Hein Plaque Index (18) was chosen because it can detect small differences in the amount of dental plaque, better demonstrating the capacity of plaque removal.

Table 1. Mean ( \pm SD) Quigley \& Hein plaque index mean before and after toothbrushing with and without use of dentifrice $(n=24)$.

\begin{tabular}{lccc}
\hline Group & $\begin{array}{c}\text { Before } \\
\text { toothbrushing }\end{array}$ & $\begin{array}{c}\text { After } \\
\text { toothbrushing }\end{array}$ & p value* \\
\hline w/ dentifrice & $3.76 \pm 0.22$ & $1.12 \pm 0.36$ & 0.01 \\
w/out dentifrice & $3.68 \pm 0.26$ & $1.16 \pm 0.31$ & 0.02 \\
p value* & 0.10 & 0.32 & \\
\hline
\end{tabular}

*Student's paired t-test.

Table 2. Mean ( \pm SD) Quigley \& Hein plaque index in palatal/lingual and buccal surfaces before and after toothbrushing with and without dentifrice $(n=24)$.

\begin{tabular}{lccccc}
\hline \multirow{2}{*}{ Group } & \multicolumn{2}{c}{ Before toothbrushing } & & \multicolumn{2}{c}{ After toothbrushing } \\
\cline { 2 - 3 } \cline { 5 - 6 } & $\begin{array}{c}\text { Lingual/ } \\
\text { palatal }\end{array}$ & Buccal & & $\begin{array}{c}\text { Lingual/ } \\
\text { Palatal }\end{array}$ & Buccal \\
\hline w/ dentifrice & $2.78 \pm 0.56$ & $3.76 \pm 0.22$ & & $1.22 \pm 0.27^{*}$ & $1.12 \pm 0.36^{*}$ \\
$\begin{array}{l}\text { w/out } \\
\text { dentifrice }\end{array}$ & $2.89 \pm 0.42$ & $3.68 \pm 0.26$ & & $1.19 \pm 0.33^{*}$ & $1.16 \pm 0.31^{*}$ \\
& & & & & \\
p value* & 0.20 & 0.10 & & 0.38 & 0.32 \\
\hline
\end{tabular}

*Intragroup significant differences before and after toothbrushing $(\mathrm{p}<0.05$, Student's paired t-test).
Accordingly, it improves the assessment sensitivity for attempting to achieve a more precise result about the remaining plaque. The period of plaque accumulation (72 h) allowed a sufficient amount of plaque to adhered to tooth surfaces. Brushing duration ( $2 \mathrm{~min}$ for the whole mouth) has been widely used to assess plaque control efficacy $(19,20)$. Additionally, the groups were matched with respect to the amount of plaque at baseline. All these features contributed to increase the validity of the study.

Using a crossover, blind and randomized methodology, Binney et al. (12) found similar results to ours, as toothbrushing with dentifrice did not have an additional benefit to plaque removal. However, that study had a smaller sample size, with only 11 participants. Important methodological aspects such, as sample size calculation and examiner calibration, were not used in that study. The present study found similar results, however, with more careful methodological considerations.

Parizotto et al. (13) also showed similar results to those of the present study in 4-6-year-old children. However, in that study, 8 children were assigned to each group under a parallel-group comparison, which increases variability and requires larger sample sizes. In addition, the children themselves brushed their teeth, which is not recommended at this age. The results of Danielsen et al. (14) were also similar to ours. They evaluated 70 individuals aged 12-16 years using a crossover design. However, the study tested the effect of chewing gum, which could have confused the outcomes. In our study, the sample comprised individuals aging more than 17 years and a splitmouth design was employed. Thus, both experimental procedures were carried out in the same participant. Variability inherent to toothbrushing technique and force should be equally distributed in the experimental groups. A spillover effect cannot be ruled out. However, the dentifrice used does not contain any particular antiplaque agent, which diminishes the possibility of a confounding bias.

The results of present study reinforces the important effect of toothbrush alone in removing dental plaque. In vivo evidence has shown that dentifrice does not enhance plaque 
removal when used in conjunction with a toothbrush $(21,22)$. According to Creeth et al. (22) plaque removal increased with brushing time. The authors showed that brushing for $180 \mathrm{~s}$ removed $55 \%$ more plaque than brushing for $30 \mathrm{~s}$ and brushing for $120 \mathrm{~s}$ removed $26 \%$ more plaque than brushing for $45 \mathrm{~s}$. Zanatta et al. (23) showed an incidence of $3 \%$ in gingival abrasion after only one time toothbrushing using soft-bristle brush without dentifrice. In addition, in vitro evidence has demonstrated that differences of toothbrushing force (24) and even in bristle flexibility of soft-classified toothbrushes (25) have demonstrated differences in hard tissue abrasion potential.

On the other hand, Eid and Talic (16) compared toothbrushing with dentifrice and with water and found that the former was more efficient in removing dental plaque. In that study, the authors used parallel groups and toothbrushing was not performed by the participants, but rather by a dentist. This is a limitation in the validity of our study. On the other hand, having toothbrushing performed by the participants themselves would reproduce closely a real situation, which might increase the external validity and enable further inferences. Almajed (15) also found a higher efficacy of plaque removal by toothbrushing with dentifrices compared with toothbrushing alone. However, the participants were kept in the experimental groups for 1 week. The results suggest that the additional effect of the dentifrices on dental plaque could be cumulative, but they could also be biased, since there was not a control over the participants.

The fact that the present study did not demonstrate significant differences between toothbrushing with and without dentifrices does not mean that brushing alone should be encouraged. It is well known that dentifrices play an important role on the prevention of caries and periodontal diseases due to their formulation, especially the presence of fluoride and antimicrobials. Moreover, this is an efficacy study in which only one toothbrushing cycle was performed, and doe not allow drawing conclusions that would include continuous use of dentifrice. Possibly, a clinical inference would be that when giving oral hygiene instructions to their patients, dentists could avoid using dentifrice in order to reinforce the message that the cleaning capacity of toothbrushing is not enhanced by the use of dentifrice. Also, the visualization of the oral hygiene procedure could make instruction and re-instruction easier. This way, the use of conventional dentifrices would be much more related to topical application of fluoride than to increasing plaque removal.

In conclusion, the use of a conventional dentifrice during toothbrushing does not seem to enhance plaque removal capacity.

\section{RESUMO}

O objetivo deste estudo foi comparar a eficácia de remoção mecânica da placa dental através da escovação com e sem dentifrício. Vinte e quatro estudantes com idade de 17 a 28 anos participaram deste ensaio clínico randomizado. Os quadrantes 1-3 ou 2-4 foram randomizados para alocação no grupo teste (escovação sem dentifrício) ou grupo controle (escovação com dentifrício). Após $72 \mathrm{~h}$ de cessação de higiene bucal, o índice de placa de Quigley \& Hein (Turesky) foi avaliado antes e após a escovação por um examinador cego e calibrado. Comparações intra e intergrupo foram realizadas pelo teste t pareado, a um nível de significância de 5\%. Os resultados demonstraram que, após a escovação, ambos os grupos apresentaram redução significativa de placa, porém sem diferenças intergrupos $(3,06 \pm 0,54$ a $1,27 \pm$ 0,26 versus $3,07 \pm 0,52$ a 1,31 $\pm 0,23$ ). Uma análise separada das faces vestibulares e linguais também não revelou diferenças entre os grupos. Conclui-se que a utilização de dentifrícios associado ao controle mecânico parece não contribuir para a remoção da placa dental.

\section{ACKNOWLEDGEMENTS}

The authors wish to thank the volunteers who participated in this research.

\section{REFERENCES}

1. Socransky SS, Haffajee AD. Dental biofilms: difficult therapeutic targets. Periodontol 2000 2002;28:12-55.

2. Löe H, Theilade E, Jensen SB. Experimental gingivitis in man. J Periodontol 1965;36:177-187.

3. Lindhe J, Hamp SE, Löe H. Plaque induced periodontal disease in beagle dogs. A 4-year clinical roentgenographical and histometrical study. J Periodontol Res 1975;10:243-255.

4. Cancro LP, Fischman SL. The expected effect on oral health of dental plaque control through mechanical removal. Periodontol 2000 1995;8:60-74.

5. Iacono VJ, Aldredge WA, Lucks H, Schwartzstein S. Modern supragingival plaque control. Int Dent J 1998;48:290-297.

6. Sheiham A. Is the chemical prevention of gingivitis necessary to prevent severe periodontitis? Periodontol 2000 1997;15:15-24.

7. West NX, Moran JM. Home-use preventive and therapeutic oral products. Periodontol 2000 2008;48:7-9.

8. Moran JM. Home-use oral hygiene products: mouthrinses. Periodontol 2000 2008;48:42-53.

9. Von der Fehr FR, Møller IJ. Caries-preventive fluoride dentifrices. Caries Res 1978;12:31-37.

10. Wülknitz P. Cleaning power and abrasivity of European toothpastes. Adv Dent Res 1997;11:576-579.

11. Guimaräes LEH, Panzeri H. Study of abrasivity of dentifrices basic formulations. Rev Cienc Farm 1998;19:22-28.

12. Binney A, Addy M, Newcombe RG. The plaque removal effects 
of single rinsings and brushings. J Periodontol 1993;64:181-185.

13. Parizotto SP, Rodrigues CR, Singer Jda M, Sef HC. Effectiveness of low cost toothbrushes, with or without dentifrice, in the removal of plaque in deciduous teeth. Pesqui Odontol Bras 2003;17:17-23.

14. Danielsen B, Baelum V, Manji F, Fejerskov O. Chewing sticks, toothpaste, and plaque removal. Acta Odontol Scand 1989;47:121125.

15. Almajed I. A comparative study between the double-headed toothbrush and the single headed toothbrush in plaque removal efficiency. J Clin Pediatr Dent 1994;19:19-21.

16. Eid MA, Talic YF. A clinical trial on the effectiveness of professional toothbrushing using dentifrice and water. Odontostomatol Trop 1991;14:9-12.

17. Quigley GA, Hein JW. Comparative cleansing efficiency of manual and power brushing. J Am Dent Assoc 1962;65:26-29.

18. Turesky S, Gilmore ND, Glickman I. Reduced plaque formation by the chloromethyl analogue of victamine C. J Periodontol 1970;41:41-43.

19. Saxer UP, Barbakow J, Yankell SL. New studies on estimated and actual toothbrushing times and dentifrice use. J Clin Dent 1998;9:49-51.
20. Attin T, Hornecker E. Tooth brushing and oral health: how frequently and when should tooth brushing be performed? Oral Health Prev Dent 2005;3:135-140.

21. Jayakumar A, Padmini H, Haritha A, Reddy KP. Role of dentifrice in plaque removal: a clinical trial. Indian J Dent Res 2010;21:213217.

22. Creeth JE, Gallagher A, Sowinski J, Bowman J, Barrett K, Lowe S, et al.. The effect of brushing time and dentifrice on dental plaque removal in vivo. J Dent Hyg 2009;83:111-116.

23. Zanatta FB, Bergoli AD, Werle SB, Antoniazzi RP. Biofilm removal and gingival abrasion with medium and soft toothbrushes. Oral Health Prev Dent 2011;9:177-183.

24. Tellefsen G, Tellefsen G, Liljeborg A, Johannsen A, Johannsen G. The role of the toothbrush in the abrasion process. Int J Dent Hyg 2011;9:284-290.

25. Teche FV, Paranhos HF, Motta MF, Zaniquelli O, Tirapelli C. Differences in abrasion capacity of four soft toothbrushes. Int $\mathrm{J}$ Dent Hyg 2011;9:274-278. 